\title{
SIGNED COMPLETE GRAPHS WITH MAXIMUM INDEX
}

\author{
SAieed Akbari \\ Department of Mathematical Science \\ Sharif University of Technology, Tehran, Iran \\ e-mail: s_akbari@sharif.edu \\ Soudabeh Dalvandi, Farideh Heydari
}

AND

Mohammad Maghasedi

Department of Mathematics

Karaj Branch, Islamic Azad University, Karaj, Iran

e-mail: s.dalvandi@kiau.ac.ir

f-heydari@kiau.ac.ir

maghasedi@kiau.ac.ir

In the Memorial of Professor Slobodan Simić

\begin{abstract}
Let $\Gamma=(G, \sigma)$ be a signed graph, where $G$ is the underlying simple graph and $\sigma: E(G) \longrightarrow\{-,+\}$ is the sign function on the edges of $G$. The adjacency matrix of a signed graph has -1 or +1 for adjacent vertices, depending on the sign of the edges. It was conjectured that if $\Gamma$ is a signed complete graph of order $n$ with $k$ negative edges, $k<n-1$ and $\Gamma$ has maximum index, then negative edges form $K_{1, k}$. In this paper, we prove this conjecture if we confine ourselves to all signed complete graphs of order $n$ whose negative edges form a tree of order $k+1$. A [1,2]-subgraph of $G$ is a graph whose components are paths and cycles. Let $\Gamma$ be a signed complete graph whose negative edges form a $[1,2]$-subgraph. We show that the eigenvalues of $\Gamma$ satisfy the following inequalities:
\end{abstract}

$$
-5 \leq \lambda_{n} \leq \cdots \leq \lambda_{2} \leq 3 .
$$

Keywords: signed graph, complete graph, index.

2010 Mathematics Subject Classification: 05C22, 05C50. 


\section{REFERENCES}

[1] N. Abreu, D.M. Cardoso, F.A.M. Franca and C.T.M. Vinagre, On main eigenvalues of certain graphs, Comput. Appl. Math., to appear.

[2] S. Akbari, S. Dalvandi, F. Heydari and M. Maghasedi, On the eigenvalues of signed complete graphs, Linear Multilinear Algebra 67 (2019) 433-441. doi:10.1080/03081087.2017.1403548

[3] S. Akbari, S. Dalvandi, F. Heydari and M. Maghasedi, On the multiplicity of -1 and 1 in signed complete graphs, Util. Math., to appear.

[4] F. Belardo, P. Petecki and J. Wang, On signed graphs whose second largest Laplacian eigenvalue does not exceed 3, Linear Multilinear Algebra 64 (2016) 1785-1799. doi:10.1080/03081087.2015.1120701

[5] A.E. Brouwer and W.H. Haemers, Spectra of Graphs (Springer, 2011). doi:10.1007/978-1-4614-1939-6

[6] D. Cvetković, P. Rowlinson and S.K. Simić, An Introduction to the Theory of Graph Spectra (Cambridge University Press, Cambridge, 2010).

[7] F. Harary, On the notion of balance in a signed graph, Michigan Math. J. 2 (1953) 143-146. doi:10.1307/mmj/1028989917

[8] F. Heider, Attitude and cognitive organization, J. Psychology 21 (1946) 107-112. doi:10.1080/00223980.1946.9917275

[9] T. Koledin and Z. Stanić, Connected signed graphs of fixed order, size, and number of negative edges with maximal index, Linear Multilinear Algebra 65 (2017) 21872198.

doi:10.1080/03081087.2016.1265480

[10] H. Lin, R. Liu and J. Shu, Some results on the largest and least eigenvalues of graphs, Electron. J. Linear Algebra 27 (2014) 670-682. doi:/10.13001/1081-3810.1928

[11] S.M. Lv, L. Wei and H.X. Zhao, On the Seidel integral complete multipartite graphs, Acta Math. Appl. Sin. Engl. Ser. 28 (2012) 705-710.

doi:10.1007/s10255-012-0126-x

[12] Z. Stanić, Perturbations in a signed graph and its index, Discuss. Math. Graph Theory 38 (2018) 841-852.

doi:10.7151/dmgt.2035

Received 4 June 2019

Revised 26 November 2019

Accepted 26 November 2019 